\title{
Efficiency in detection of isoluminant and isochromatic interference fringes
}

\author{
Nobutoshi Sekiguchi, ${ }^{*}$ David R. Williams, and David H. Brainard ${ }^{+}$ \\ Center for Visual Science, University of Rochester, Rochester, New York 14627
}

Received August 19, 1992; revised manuscript received April 7, 1993; accepted April 8, 1993

\begin{abstract}
We examined the limitations imposed by neural factors on spatial contrast sensitivity for both isochromatic and isoluminant gratings. We used two strategies to isolate these neural factors. First, we eliminated the effect of blurring by the dioptrics of the eye by using interference fringes. Second, we corrected our data for additional sensitivity losses up to and including the site of photon absorption by applying an ideal-observer analysis described by Geisler [J. Opt. Soc. Am. A 1, 775 (1984)]. Our measurements indicate that the neural visual system modifies the shape of the contrast-sensitivity functions for both isochromatic and isoluminant stimuli at high spatial frequencies. If we assume that the high-spatial-frequency performance of the neural visual system is determined by a low-pass spatial filter followed by additive noise, then the visual system has a spatial bandwidth 1.8 times lower for isoluminant red-green than for isochromatic stimuli. On the other hand, we find no difference in bandwidth or sensitivity of the neural visual system for isoluminant red-green and S-coneisolated stimuli.
\end{abstract}

\section{INTRODUCTION}

The efficiency with which the visual system processes luminance and chromatic information has been measured with a number of different tasks that depend on stereopsis, ${ }^{1-3}$ temporal modulation sensitivity, ${ }^{4,5}$ motion perception, ${ }^{6-8}$ accommodation, ${ }^{9,10}$ border perception, ${ }^{11,12}$ vernier acuity, ${ }^{13,14}$ and spatial contrast sensitivity. ${ }^{15-17}$ For separating the processing of luminance and chromatic information, isochromatic and isoluminant stimuli typically are used. Isochromatic stimuli are modulated in luminance but are uniform in chromaticity. Conversely, isoluminant stimuli are modulated in chromaticity but are uniform in luminance. Generally, performance is better for isochromatic than for isoluminant stimuli, at least when the task requires high-spatial-frequency information. The origin of this performance difference is unclear. Differences in sensitivity between isochromatic and isoluminant stimuli can be caused by effects of the dioptrics of the eye (e.g., diffraction and chromatic aberration), by optical factors of the cone mosaic (e.g., cone aperture, spatial sampling, and photopigment spectral responsivities), or by subsequent neural factors. In this paper we examine the limitations imposed by neural factors on spatial contrast sensitivity for both isochromatic and isoluminant gratings. We used two strategies to isolate these neural factors. First, by using interference fringes, we eliminated the effect of blurring by the eye's optics. Second, we corrected our data for sensitivity losses up to and including the site of photon absorption by applying an ideal-observer analysis. ${ }^{18-23}$ This paper provides estimates of the spatial bandwidth of the neural visual system for three different directions in color space: isochromatic, red-green isoluminant, and shortwavelength-sensitive- (S-) cone-isolated modulations.

\section{Eliminating the Effects of the Eye's Optics}

Measurement of red-green-isoluminant contrast sensitivity is complicated by the effects of axial and transverse chromatic aberration, both of which can contaminate the measurements by introducing a luminance artifact. ${ }^{24,25}$ We have developed a new apparatus and psychophysical technique for measuring red-green-isoluminant contrast sensitivity that minimizes the possibility of such contamination. By using interference fringes, we eliminated the possibility of a luminance artifact caused by axial chromatic aberration. We eliminated the possibility' of a luminance artifact caused by transverse chromatic aberration by employing a new psychophysical technique in which equally luminant red and green interference fringes were drifted in opposite directions. A detailed treatment of our apparatus and technique is provided in the companion paper. ${ }^{26}$

More generally, the use of interference fringes avoids blurring by the eye's optics. This provides two important advantages for the purposes of this paper. First, to compute neural efficiency it is necessary to compare realand ideal-observer performance. By avoiding blurring by the optics of our real observers, we avoid uncertainty in this comparison that would otherwise arise because the effects of the eye's optics are not entirely quantified, individual differences in optical quality exist, ${ }^{27}$ and fluctuations in accommodation can affect performance even within a single individual. Second, avoiding blurring allows us to extend our red-green-isoluminant contrastsensitivity measurements to $20-27$ cycles per degree (c/deg), depending on the observer, substantially higher than has been measured previously. ${ }^{15-17,28-33}$ This allows us to compare performance for isochromatic and isoluminant stimuli at high spatial frequencies, which is the focus of this paper.

\section{Computing Neural Efficiency}

We estimate neural efficiency by comparing the spatial contrast sensitivity of real and ideal observers under similar stimulus conditions. Our ideal observer incorporates sensitivity losses that are due to optical factors up to and 
including photopigment absorption. Note that by optical factors we refer to effects of the photon noise, the anterior optics of the eye (e.g., cornea, lens, and ocular media), and the photoreceptor mosaic (e.g., cone aperture, spatial sampling, and photopigment spectral responsivities). We will refer to all subsequent factors as neural factors. We attribute differences between real and ideal observers to neural stages that follow photopigment absorption. In essence, the ideal observer reveals the impact of optical factors so that subsequent neural factors can be assessed independently.

We illustrate the value of this approach by describing two specific hypotheses that we are poised to test. First, consider the effect of the overlapping spectral responsivity of the long-wavelength-sensitive (L) and the middlewavelength-sensitive (M) cones. This overlap ensures that the highest physically realizable isoluminant contrast does not modulate the cones as much as does a $100 \%$ contrast isochromatic modulation. It has been suggested that if this overlap were taken into account, then the subsequent neural visual system might be equally efficient at detecting isochromatic and isoluminant gratings. ${ }^{5}$ A comparison of real and ideal observer performance for the same stimuli would permit a proper test of this hypothesis. ${ }^{18,19}$ Previous attempts at such a comparison have been limited by uncertainty about optical blurring and by the limited spatial-frequency range of existing isoluminant contrast-sensitivity data. The data we present in this paper overcome these limitations. As a second example, Banks et $a ._{.22}$ argued that the shape of the foveal contrastsensitivity function for isochromatic spatial modulations can be predicted without consideration of the properties of the neural visual system. We also examine this hypothesis with data that avoid uncertainty about optical blurring.

Next we describe the construction and performance of our ideal observer. Following that, we present corresponding psychophysical methods used with real observers and the results obtained. Together the two analyses allow us to compute neural efficiency for the detection of gratings modulated in different directions in color space.

\section{PERFORMANCE OF THE IDEAL OBSERVER}

\section{Methods}

Overview. Our ideal observer is similar to the one developed by Geisler..$^{18,20}$ The main difference between our analysis and Geisler's is that our ideal observer does not include blurring by the dioptrics of the eye. To compute ideal-observer performance, we simulate the performance of an ideal decision maker on a series of two-interval forced-choice trials. The ideal decision maker has access to the output of a model visual system that incorporates our best estimates of the optical factors that affect realobserver performance. Figure 1 shows the various stages of our ideal observer. Our simulation allows us to determine ideal-observer contrast sensitivity for gratings of different spatial frequencies modulated in different directions in color space.

Stimuli. Ideal-observer performance was computed for three types of grating stimulus, as shown in Fig. 2. These gratings correspond to an isochromatic grating, a redgreen isoluminant grating, and an S-cone-isolating grating. The spectral compositions and retinal illuminances were the same as those used in our psychophysical experiments and are described later in this paper. For each type of grating we computed ideal-observer contrast sensitivity at a series of spatial frequencies. At each spatial frequency, contrast threshold corresponded to $75 \%$-correct performance for the ideal observer on a two-interval forced-choice task. One interval contained the grating stimulus, and the second interval contained an identical field, except that it had no modulation at the grating frequency. Across spatial frequencies, the size of the grating patch was scaled so that 5 grating cycles were contained within 4 standard deviations of the Gaussian window.

Photon noise. Each stimulus was degraded by photon noise. This is the only source of noise in the ideal observer.

Ocular transmittance. We interpolated the data of Wyszecki and Stiles ${ }^{34}$ on lens and macular pigment absorption in the average young eye for the specific wavelengths used in the psychophysical measurements discussed later in this paper. The ocular transmittance for the 441.6-, 514.5-, 580.0-, and 632.8-nm lights used were $12 \%, 40 \%$, $58 \%$, and $62 \%$, respectively.

Cone aperture. We defined the aperture size (the full width at half-height) of each cone as $34 \%$ of the cone spacing. The cone-aperture size affects the shape of the contrast-sensitivity function for isoluminant and isochromatic stimuli in the same way. The cones function as waveguides, ${ }^{35}$ and the efficiency of the photon capture in space can be approximated by a Gaussian function. MacLeod et $a l .^{36}$ provided psychophysical estimates of the cone aperture, estimating that the full width at half-

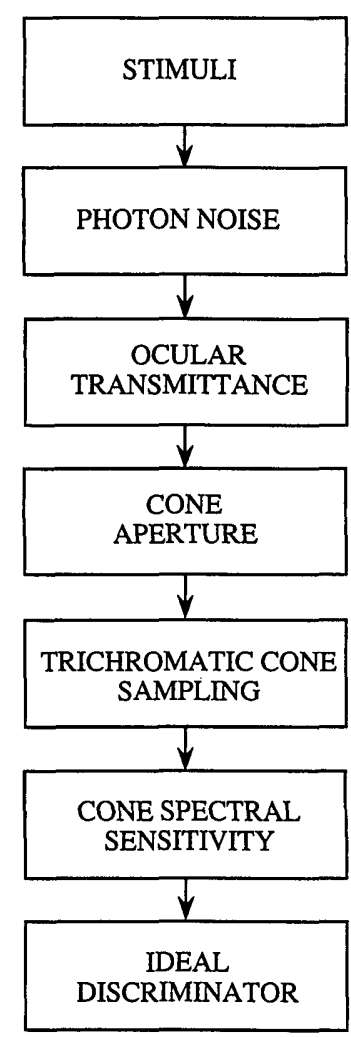

Fig. 1. Structure of our ideal observer. The output of the model visual system is computed by passing the stimulus, degraded by photon noise, through a series of stages. Each stage incorporates one factor known to affect real-observer visual performance. 
(a)

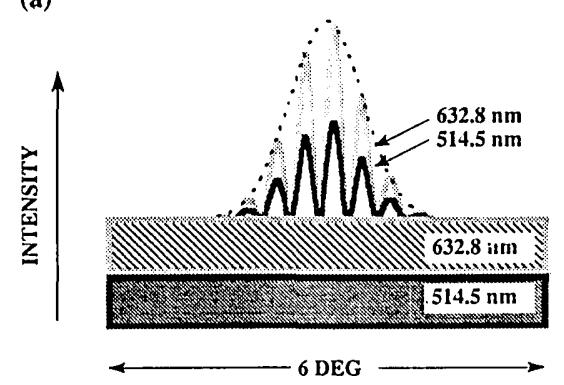

(b)

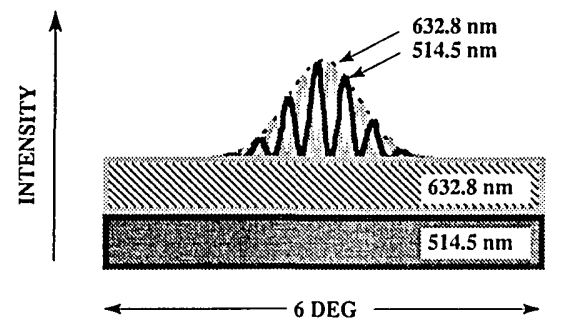

(c)

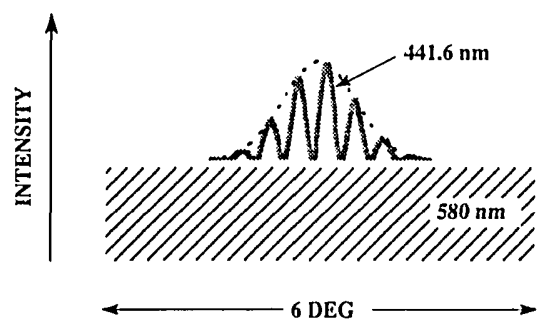

Fig. 2. Luminance profile of (a) the isochromatic, (b) the isoluminant red-green, and (c) the S-cone-isolating gratings.

height of the Gaussian is 0.225 arcmin (the mean of three observers). We determined the ratio of the cone aperture diameter to the cone spacing by assuming that their result reflects the aperture size of the cones that are most closely packed (0.66 arcmin in center-to-center spacing). For parafoveal cones, the presence of rods in the parafoveal region may cause this rule to overestimate the coneaperture size. However, the proportion of area occupied by rods is very small $(\sim 3.3 \%)$, and this does not produce a significant difference in the contrast-sensitivity estimates.

Trichromatic cone sampling. We used two models of the cone mosaic, as shown in Fig. 3. Figure 3(a) is a model from the central $1 \mathrm{deg}$ of the monkey fovea, provided by Hugh Perry. Figure $3(\mathrm{~b})$ is a model from a parafoveal human cone mosaic patch (approximately 1-deg-square patch centered at $\sim 1 \mathrm{deg}$ in the temporal retina), provided by Christine Circio (see Ref. 37). We used two cone mosaics to correspond to the two retinal locations at which our psychophysical data were obtained. We used a parafoveal location in addition to a foveal location because it avoids the tritanopic area ${ }^{38}$ at the foveal center and corresponds to the region where S-cone density is highest. ${ }^{37}$ Each cone mosaic model consists of a number of dots, each of which represents the center of one cone. Applying the Delaunay triangulation procedure, we estimated the center-to-center cone spacing of these cone mosaic models.
(We used the Delaunay triangulation software kindly provided by Ken Sloan of University of Alabama.) For the foveal cone mosaic, cone spacing ranges between 0.66 and $1.2 \mathrm{~min}$, and the mean cone spacing in the central 20 -min area is $0.75 \mathrm{~min}$. Because of the smaller eye of the monkey, these estimates of cone spacing are approximately 1.3 times larger than the spacing that is typical of the human fovea. However, cone spacing itself is not a critical factor in determining contrast sensitivity of the ideal observer, a point to which we return below. For the parafoveal cone mosaic, cone spacing varies less than in the fovea. The mean cone spacing across the whole mosaic is $1.1 \mathrm{~min}$.

We assumed that the relative population of $\mathrm{S}$ cones in the human fovea is approximately $5 \%$, after Curcio et $a l^{37}$ However, there remains some uncertainty about the relative numbers of the $\mathrm{L}$ and $\mathrm{M}$ cones ${ }^{39-43}$ and their packing geometry. In this study we have assumed that the ratio of $\mathrm{L}$ to $\mathrm{M}$ cones is $2: 1$, although this assumption is not particularly critical. In this study the three cone types were assigned randomly in both foveal and parafoveal cone mosaic models. The foveal model contained a tritanopic area $20 \mathrm{~min}$ in diameter centered on the location of highest cone density. ${ }^{37,38}$

Photopigment spectral responsivities. The absorption spectra used for the three cone types were those obtained

(a)

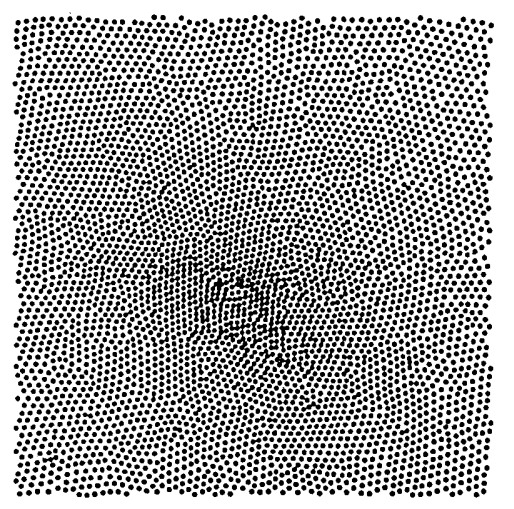

FOVEA

(b)

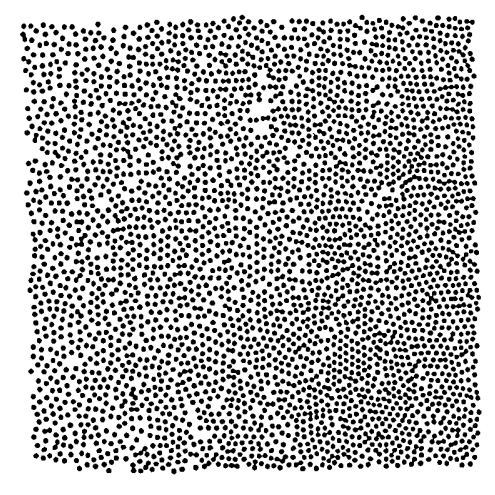

\section{DEG TEMPORAL}

Fig. 3. Cone mosaic models used in this study: (a) the central 1 deg of monkey fovea, (b) the parafoveal human cone mosaic from Curcio et al. ${ }^{37}$ 

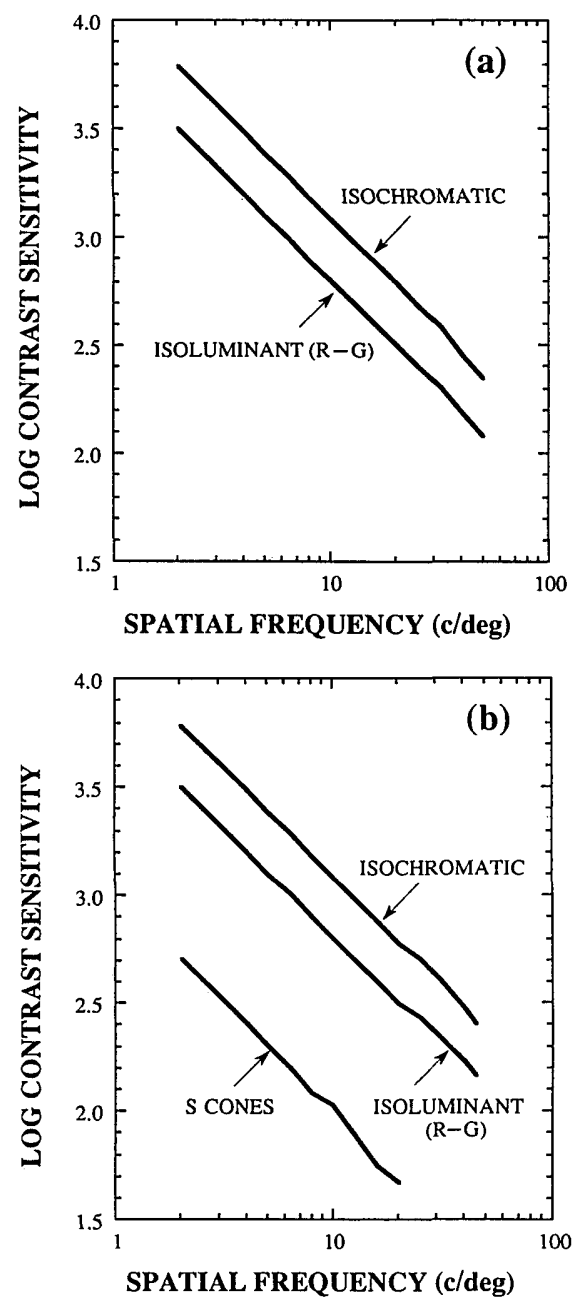

Fig. 4. Ideal observer's contrast-sensitivity functions at (a) the fovea and (b) the parafovea.

with the suction electrode technique. ${ }^{44}$ We assumed that the pigment density of each cone type was set at $0.44 \mathrm{log}$ unit at the wavelength of its peak responsivity.

Ideal discriminator. The ideal discriminator was a maximum-likelihood decision rule identical to that used by Geisler. ${ }^{18,20}$ The ideal discriminator had access to the number of photopigment absorptions in each cone. The discriminator had exact knowledge of the distribution of the number of these photopigment absorptions for each stimulus.

\section{Results}

Figures 4(a) and 4(b) show the ideal observer's contrastsensitivity functions computed with the foveal and the parafoveal cone mosaic models, respectively. Vertical axes represent the reciprocal of the contrast of the component gratings that produce $75 \%$ correct response.

Slopes of the contrast-sensitivity functions. There are two factors that affect the slopes of the ideal observer's contrast-sensitivity functions. These are blurring by the cone aperture and the fact that size of the grating patch was inversely proportional to spatial frequency. (Recall that our ideal observer views interference fringes so that there is no blurring by the dioptrics of the eye.) All the contrast-sensitivity functions have slopes close to -1 on the $\log -\log$ plot. For grating stimuli with a fixed number of cycles, the total number of photons reaching the receptor mosaic decreases as the square of the spatial frequency. It may be shown analytically that contrast sensitivity is proportional to the square root of the total number of photons absorbed. Thus, if the size of the grating patch were the only factor, the slopes of the contrastsensitivity functions would be exactly $-10^{18,22}$ The actual slope is slightly greater than -1 as a result of blurring by the cone aperture. The cone aperture reduces the effective contrast, resulting in a modest further decline in contrast sensitivity with increasing spatial frequency. Although the blurring by the cone aperture increases with its diameter, this difference is very small for our foveal and parafoveal locations. The slopes of the foveal and parafoveal curves depart appreciably only at high spatial frequencies beyond the range of interest here. In the plot, ideal-observer performance at spatial frequencies lower than $6.3 \mathrm{c} / \mathrm{deg}$ was extrapolated with a slope of -1 because the computations were thwarted by the limited size of our model cone mosaics.

\section{Effect of Color Direction in Ideal-Observer Contrast Sensitivity}

The effect of changing the color direction of the stimulus is to shift the ideal observer's contrast-sensitivity functions on the log-log plots vertically without changing their slopes. The lower sensitivity of the ideal observer to redgreen isoluminant stimuli relative to isochromatic stimuli is caused almost completely by the overlap of the spectral responsivities of the $\mathrm{L}$ and $\mathrm{M}$ cones. Our simulations indicate that neither the relative numbers of $\mathrm{L}$ and $\mathrm{M}$ cones nor their packing geometry contributes substantially to the shift. ${ }^{45}$ This is because neither of these factors has a large influence on the total number of photopigment absorptions for either type of grating.

It has been suggested that the properties of the cone mosaic necessarily imply that the slope of the red-green isoluminant contrast-sensitivity function must be steeper than the slope of the isochromatic contrast-sensitivity function. ${ }^{33}$ Our ideal-observer calculations, however, agree with those of Geisler and indicate that any difference in the slopes of real-observer contrast-sensitivity functions must be due to neural factors. ${ }^{18}$ The intuition behind this result is as follows. For both isochromatic and isoluminant gratings, the ideal observer makes optimal use of the information in both the L- and the M-cone submosaics. There are two differences in the way that isochromatic and red-green isoluminant gratings stimulate the two submosaics. First, as noted above, the contrast seen by each submosaic is generally lower for isoluminant than for isochromatic gratings. This difference is independent of spatial frequency and explains the overall vertical shift between the ideal observer's contrastsensitivity functions. The only other difference in the way that the stimuli affect the output of the mosaic is that the modulations seen by the L- and the M-cone submosaics have different spatial phase relationships for the two types of grating. The ideal observer has exact information about the stimulus, and its performance is not affected by this change in spatial phase.

The ideal observer's S-cone-isolated contrast-sensitivity function is shifted even lower than the red-green isoluminant contrast-sensitivity function, again without change in shape. The spectral responsivity of the $S$ cones is sufficiently separate from that of the $L$ and the $M$ cones 
that the overlap in spectral responsivities plays only a minor role in this shift. The reduced sensitivity to Scone stimuli can be explained almost entirely by the decrease in the number of photopigment absorptions that are due to the low density of the $\mathrm{S}$ cones compared with the density of the $\mathrm{L}$ and the $\mathrm{M}$ cones ${ }^{18}$ and the effective contrast ( $\sim 50 \%$ at maximum) of the $S$ cones obtained from the S-cone stimulus. Note that the lowered sampling density of the $\mathrm{S}$ cones does not influence the shape of the ideal observer's contrast-sensitivity function. The fact that the stimulus is sampled sparsely by the $\mathrm{S}$ cones would affect the ability of the ideal observer to identify the spatial structure of fine gratings, as it does in real observers ${ }^{46,47}$ but is not relevant to the detection task.

For different color directions, the vertical shifts in contrast sensitivity depend on the spectral composition of the stimuli. For our psychophysical measurements, in which the red-green isoluminant stimuli were produced with 632.8- and 514.5-nm gratings, the loss in sensitivity is $\sim 0.28 \log$ unit relative to that for isochromatic stimuli generated with the same gratings. Our S-cone stimuli were produced with a $441.6-\mathrm{nm}$ grating superimposed upon a 580-nm background, which results in a loss of $\sim 1.05$ log units relative to our isochromatic stimuli. The shifts in ideal-observer contrast sensitivity with the color direction of the stimuli provide a quantitative measure of the difference in contrast sensitivity that we should expect even when there is no difference in the neural efficiency with which the stimuli are detected. Following Jordan et $a l .{ }^{19}$ we will apply these shifts to the contrast sensitivity data of real observers, allowing us to compare performance for isoluminant and isochromatic stimuli on a single ordinate.

\section{PERFORMANCE OF REAL OBSERVERS}

\section{General Methods}

Isochromatic and red-green isoluminant fringes. As described in detail in the companion paper, ${ }^{26}$ we have developed a new apparatus and psychophysical technique to extend red-green isoluminant contrast-sensitivity measurements to high spatial frequencies. The apparatus consists of two identical laser interferometers that are designed to produce phase-locked two-color interference fringes on the retina without the influence of diffraction and most aberrations in the eye. We formed a grating by superimposing red $(632.8-\mathrm{nm})$ and green $(514.5-\mathrm{nm})$ interference fringes of equal spatial frequency, orientation, and space-averaged retinal illuminance. The space-averaged retinal illuminances of the two interference fringes were equated for each observer by flicker photometry. We presented all fringes in a circular field windowed by a Gaussian transparency ${ }^{26}$ to eliminate the possibility of an edge artifact. ${ }^{48,49}$ The fringes were superimposed upon a spatially uniform incoherent background 6 deg in diameter. The background was the superposition of two monochromatic fields of the same wavelengths as the fringes. The retinal illuminances of the two background components were equal. The purpose of the incoherent background was to reduce the influence of speckle masking. ${ }^{50}$ In most of the measurements the proportion of the total retinal illuminance that was coherent light, which we refer to as the coherent fraction, was $9 \%$. For the higher spatial frequencies, where contrast sensitivity is low, the coherent fraction was $48 \%$.

At the beginning of each session, the contrast of both fringes was set to zero and the observer adapted to the test field for $1 \mathrm{~min}$. The red and the green fringes were then made to drift slowly in opposite directions at $0.25 \mathrm{~Hz}$. Drifting of the two fringes guarantees that both a purely isochromatic and a purely isoluminant stimulus will be presented during each cycle of stimulus presentation despite small movements of the observer's head and eyes. The spatial profiles and the wavelength composition of the stimulus for the isochromatic and red-green isoluminant fringe phases are shown in Fig. 2. We developed and used this drifting technique to avoid contamination of our measurements by luminance artifacts, as discussed in the companion paper. ${ }^{26}$ The contrasts of the two fringes were equal, and the observer adjusted the contrasts of both fringes simultaneously to find threshold. To measure isochromatic contrast sensitivity, the observer adjusted the contrast until the luminance modulation was just detectable. To measure isoluminant contrast sensitivity, the observer adjusted contrast until the red and green modulation was just detectable.

The observer could have used either of two subjectively distinct criteria to set contrast threshold for isoluminant stimuli. Consider the case at high spatial frequencies, where the isochromatic modulation of the stimulus is more salient than the isoluminant modulation at any stimulus contrast. Either the observer could have adjusted the contrast so that there was a very brief time during each stimulus period when no modulation whatsoever was visible (when the red and the green gratings were out of phase) or he could have adjusted the contrast until no chromatic stripes were visible at any time during each stimulus period. Usually observers used the latter criterion. However, control experiments showed that these two criteria produced estimates of contrast sensitivity that were not significantly different, suggesting that the isoluminant contrast-sensitivity measurements are not contaminated by luminance artifacts or aliasing effects. We also validated the technique by showing that the contrast-sensitivity estimates that it produces are similar to those obtained with conventional static fringe stimuli and forced-choice methods. ${ }^{26}$ Typically, the observer first made four settings for isochromatic contrast threshold and then made another four settings for isoluminant contrast threshold. For each condition, at least eight settings were made in two or three sessions on different days.

We used this technique at two retinal locations: the fovea and $1 \mathrm{deg}$ in the temporal retina. The flickerphotometric measurements were always made at the site of stimulus presentation. The adjustment of the fringe spatial frequency and orientation that was required for the fringes to be brought into register ${ }^{26}$ was made in the fovea to increase the accuracy.

$S$-cone isolated fringes. The S-cone isolated stimulus was similar to the red-green stimulus in both spatial and temporal factors. It consisted of a violet (441.6-nm) interference fringe superimposed upon an incoherent yellow (580-nm) background, as shown in Fig. 2. We produced the 441.6-nm fringe by using one of the two interferometers but with a He-Cd laser source. The violet fringe was presented in a circular field windowed by a Gaussian 
transparency. ${ }^{26}$ The yellow background was a spatially uniform circular field of 6-deg diameter, and its radiance was set at 84000 trolands (Td) (11.1 log quanta $\left.\mathrm{sec}^{-1} \mathrm{deg}^{-2}\right)$. The purpose of the intense yellow background was to isolate the $S$ cones. ${ }^{46,47}$

A fixation mark, which appears as a dark cross, was presented on the background field so that the violet fringe lay at $1 \mathrm{deg}$ in the temporal retina. We chose this location to avoid the influence of the tritanopic area and to center the stimulus on the retinal location with the highest S-cone density. ${ }^{37,51-53}$

Before the measurements, the observer adapted for 2 min to the background and test field, which was temporarily set at zero contrast. To equate the temporal factors of the S-cone stimuli to those of the red-green stimuli, we modulated the contrast of the violet fringe sinusoidally in counterphase at $0.5 \mathrm{~Hz}$. The emergence of the peak contrast was marked by a short tone. Contrast threshold was measured by the method of adjustment, and the observer made four settings of contrast threshold for each spatial frequency. In a single session all spatial frequencies to be tested were presented in random order.

\section{EXPERIMENT 1: EFFECT OF RETINAL ILLUMINANCE}

The efficiency of the neural visual system for detecting gratings is known to depend on a number of stimulus parameters other than spatial frequency and color direction of the grating. Following Banks et al. ${ }^{22}$ we have adopted the approach of choosing values for these parameters to maximize the efficiency of the neural visual system. Experiment 1 examines the effect of retinal illuminance on real-observer contrast sensitivity for all three color directions. This calls for obtaining measurements at retinal illuminances for which the visual system obeys the de Vries-Rose square-root law. In addition, using such illuminances ensures that differences in efficiency for detecting fringes in different color directions cannot be attributed to retinal illuminances. For example, if we chose a retinal illuminance for isochromatic measurements that was in the Weber region, in which the contrast sensitivity was constant regardless of retinal illuminance while the S-cone-isolated measurements were in the square-root-law region, the difference in the vertical positions of the curves would be distorted by the effect of retinal illuminance. The goal of experiment 1 is to establish retinal illuminances for which detection obeys the squareroot law for all three color directions. We made the measurements at spatial frequencies within the range of interest.

\section{Methods}

Foveal contrast sensitivities for isochromatic and redgreen isoluminant stimuli were measured as a function of retinal illuminance. The fringe spatial frequency was set at $10 \mathrm{c} / \mathrm{deg}$. The test stimuli were presented at the fovea with a coherent fraction of $9 \%$. Five retinal illuminance levels were tested: $100,250,500,1000$, and $2000 \mathrm{Td}$.

Parafoveal contrast sensitivities for isochromatic, redgreen isoluminant, and S-cone-isolated stimuli also were measured. The red-green isoluminant measurements were made at $8 \mathrm{c} / \mathrm{deg}$ with a coherent fraction of $48 \%$.
The S-cone-isolated measurements 2 and $5 \mathrm{c} / \mathrm{deg}$. Four retinal illuminance levels were tested for isochromatic and red-green isoluminant stimuli: 100, 250, 500, and $1000 \mathrm{Td}$. Four retinal illuminance levels were tested for the S-cone-isolated stimuli at $2 \mathrm{c} / \mathrm{deg}$, and two levels were tested at $5 \mathrm{c} / \mathrm{deg}$; the highest level in this series was the maximum intensity obtainable with our apparatus, $8.0 \mathrm{log}$ quanta $\mathrm{sec}^{-1} \mathrm{deg}^{-2}(441.6 \mathrm{~nm})$ and $11.1 \mathrm{log}$ quanta $\sec ^{-1} \operatorname{deg}^{-2}(580 \mathrm{~nm})$

For both foveal and parafoveal measurements, the intensity series was produced by placement of neutral-density filters right before the final Maxwellian lens, which changed the total retinal illuminance level while keeping the proportions of various stimulus components constant. The test stimulus always contained 5 cycles of the fringe within 4 standard deviations of the Gaussian window, and retinal illuminance is specified for the center of the Gaussian window.

\section{Results}

The results from observer NS are shown in Fig. 5 for the fovea and in Fig. 6 for the parafovea. The horizontal axes represent the retinal illuminance measured at the center of the Gaussian envelope. The vertical axes represent contrast sensitivity. Filled and open circles show the results for the isoluminant and isochromatic stimuli, respectively. Diamonds [Fig. 6(b)] show the results for S-cone-isolated stimuli. The error bars represent \pm 1 standard error of the mean of eight settings. For the foveal isochromatic stimuli, contrast sensitivity increases with retinal illuminance, and the slope becomes shallower at $\sim 1000 \mathrm{Td}$. This finding is consistent with those of earlier studies. ${ }^{22,54,55}$ The solid lines in these figures have a slope of 0.5 on a $\log -\log$ plot, which represents the way in which ideal-observer performance increases with retinal illuminance. This is the familiar de Vries-Rose squareroot law. The lines has been vertically shifted by the eye to fit the psychophysical data. For all color directions at both retinal locations the data clearly show regions where sensitivity obeys the square-root law. In the remaining experiments we used a retinal illuminance (at the center of the Gaussian envelope) of $500 \mathrm{Td}$ for the isochromatic and the red-green isoluminant measurements and $2.6 \mathrm{Td}$ (8.0 log quanta $\mathrm{sec}^{-1} \mathrm{deg}^{-2}$ ) for the S-cone-isolated measurements.

\section{EXPERIMENT 2: EFFECTS OF GRATING SUMMATION AREA}

Experiment 2 measures the grating summation area for all three color directions. Because we wanted to compare real and ideal performance using stimulus parameters that maximized the real observer's efficiency, we chose the sizes of the grating patches to be smaller than the grating summation area for our main measurements. In addition, using such grating sizes ensures that differences in efficiency for detecting fringes in different color directions cannot be attributed to differences in grating summation for different color directions.

\section{Methods}

Contrast sensitivity for red-green-isoluminant, isochromatic, and the S-cone-isolated interference fringes was 


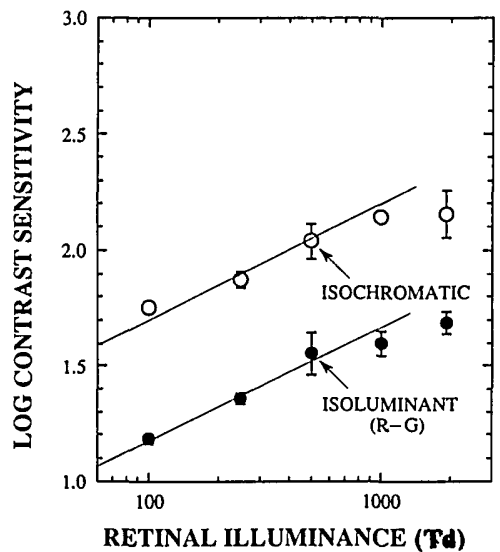

Fig. 5. Foveal isoluminant and isochromatic contrast sensitivities plotted as a function of retinal illuminance for observer NS. The solid lines represent a slope of 0.5 as predicted by the ideal observer. Each line is shifted vertically by eye to fit the psychophysical data.

measured as a function of the number of fringe cycles. The stimuli were windowed by a Gaussian, and the number of fringe cycles reported is the number of cycles contained within 4 standard deviations (approximately 1.7 times the full width at half-height) of the Gaussian window. The fringe spatial frequency was set at 4,8 , or $16 \mathrm{c} / \mathrm{deg}$ for the fovea and at 4 or $8 \mathrm{c} / \mathrm{deg}$ for $1 \mathrm{deg}$ in the temporal retina. For the S-cone stimuli, the fringe spatial frequency was set at $4 \mathrm{c} / \mathrm{deg}$. Two color-normal persons (OP and NS) served as the observers. For observer OP, only $8 \mathrm{c} / \mathrm{deg}$ was tested for the red-green conditions.

\section{Results}

Figure 7 shows the foveal results, and Fig. 8 shows the parafoveal results. Horizontal axes represent the number of fringe cycles, and vertical axes represent contrast sensitivity. The solid lines in all panels show a slope of 1 on a log-log plot that represents the performance of the ideal observer. ${ }^{18}$ The lines have been shifted vertically by eye to fit the psychophysical data. Taken as a whole, the results indicate that the grating summation area for both observers is at least 5 cycles within 4 standard deviations of the Gaussian window for all color directions at both retinal locations. Therefore we used gratings containing 5 cycles in all the subsequent experiments.

Having now established appropriate retinal illuminances and fringe sizes, we are poised to obtain contrastsensitivity measurements on real observers that can be compared with those of the ideal observer.

\section{EXPERIMENT 3: FOVEAL CONTRAST-SENSITIVITY FUNCTIONS}

\section{Methods}

We measured contrast-sensitivity functions in the fovea for isochromatic and red-green-isoluminant fringes. In a single session all spatial frequencies to be tested were presented in random order. One experimental session lasted 2-3 h, including three or four intermissions of approximately $10 \mathrm{~min}$. At least two sessions were run on different days for each observer. Three normal trichro- mats (OP, SG, and NS) served as observers. Two of them (OP and NS) had considerable experience in psychophysical experiments with interference fringes.

For each observer we used a coherent fraction of $9 \%$ for low to middle spatial frequencies and $48 \%$ for high spatial frequencies. The coherent fraction determines the highest-possible grating contrast. The higher coherent fraction was required at high spatial frequencies because of the observers' low contrast sensitivity. Williams $\mathrm{s}^{50}$ studied the effect of masking by laser speckle on isochromatic gratings. His results suggest that the present data obtained with a coherent fraction of $48 \%$ probably are affected by speckle masking, whereas those with $9 \%$ coherent fraction are not. Following Williams's procedure, we measured the effects of speckle masking by measuring the ratio of contrast sensitivities for coherent fractions of $9 \%$ and $48 \%$ at a single spatial frequency. The measured ratios were similar to those reported by Williams ${ }^{50}$ and varied between 0.07 and 0.35 log unit for different observers and color directions, but there was no systematic pattern to this variation. We corrected for the effect of
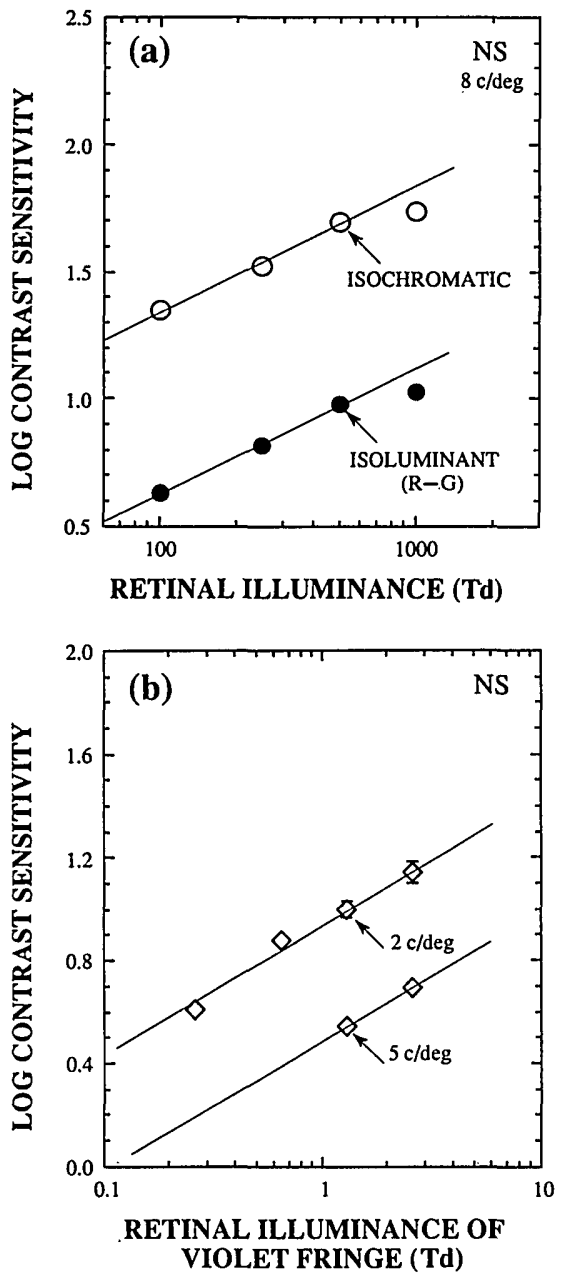

Fig. 6. (a) Parafoveal red-green isoluminant and isochromatic contrast sensitivities plotted as a function of retinal illuminance for observer NS. The solid lines represent a slope of 0.5 as predicted by the ideal observer. (b) S-cone contrast sensitivities plotted as a function of retinal illuminance. The solid lines represent a slope of 0.5 as predicted by the ideal observer. Each line is shifted vertically by eye to fit the psychophysical data. 

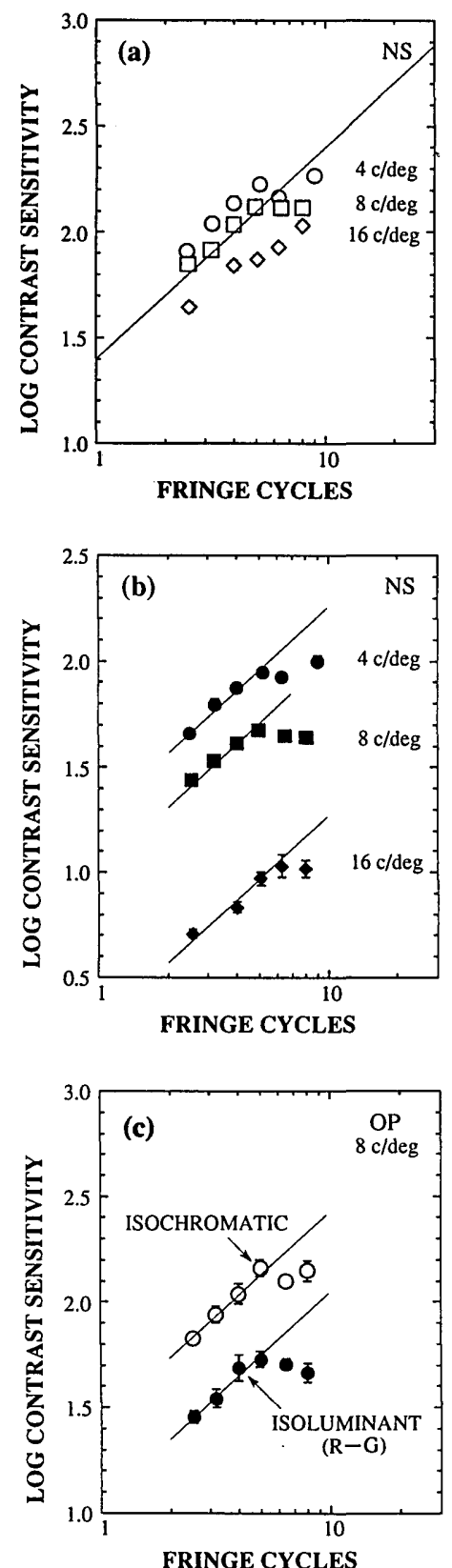

Fig. 7. Foveal contrast sensitivity for (a) isochromatic and (b) isoluminant interference fringes as a function of the number of fringe cycles for observer NS and (c) for observer OP. The solid lines have a slope of 1 on a log-log plot that is predicted by the ideal observer.

speckle masking for each observer by multiplying the sensitivities obtained with the coherent fraction of $48 \%$ by this measured ratio. This method of compensating for speckle masking is based on the assumption that speckle masking is constant with spatial frequency. This assumption has been tested for isochromatic gratings, for which it was found to be approximately true over the range of spatial frequencies examined here. ${ }^{50}$

\section{Results}

The top panels of Fig. 9 show foveal isochromatic and redgreen isoluminant contrast-sensitivity functions for three observers. The data from the different observers are similar. Filled symbols show the red-green-isoluminant contrast-sensitivity functions with its familiar low-pass shape. Open circles show the isochromatic contrastsensitivity functions, which are bandpass, as numerous previous studies have shown. Error bars represent the \pm 1 standard error of measurement, although most of them are smaller than the symbols. The filled and the open arrows in each panel represent the resolution limits for the isoluminant and the isochromatic modulations, respectively, as described in the companion paper. ${ }^{26}$
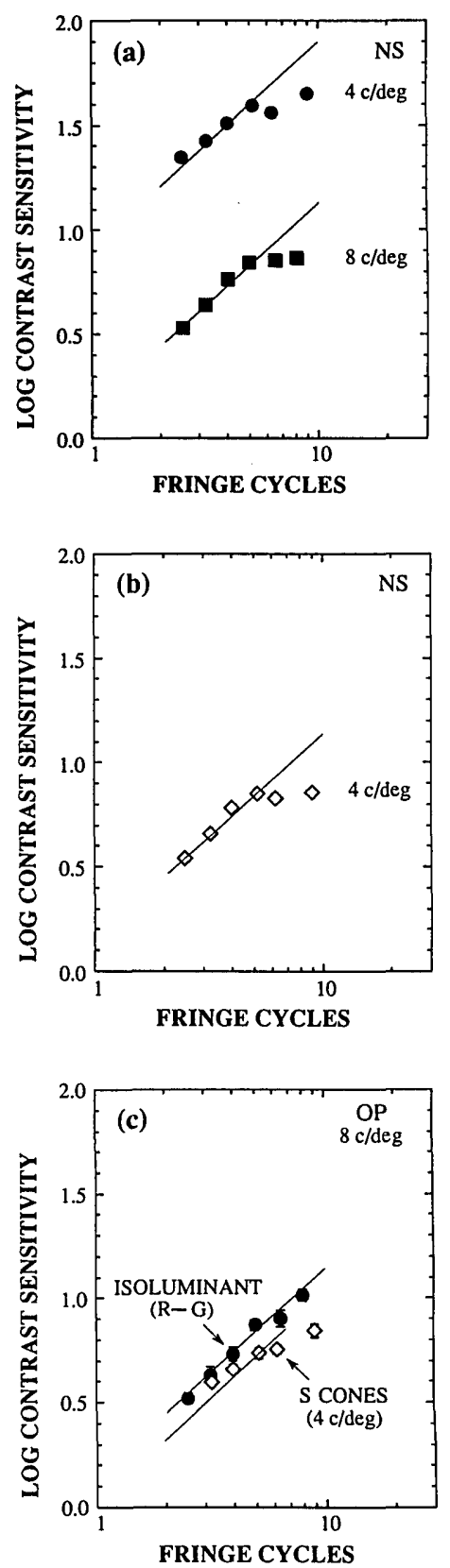

Fig. 8. Parafoveal contrast sensitivity for (a) isochromatic and (b) isoluminant interference fringes as a function of the number of fringe cycles for observer NS and (c) for observer OP. The solid lines have a slope of 1 on a log-log plot that is predicted by the ideal observer. 

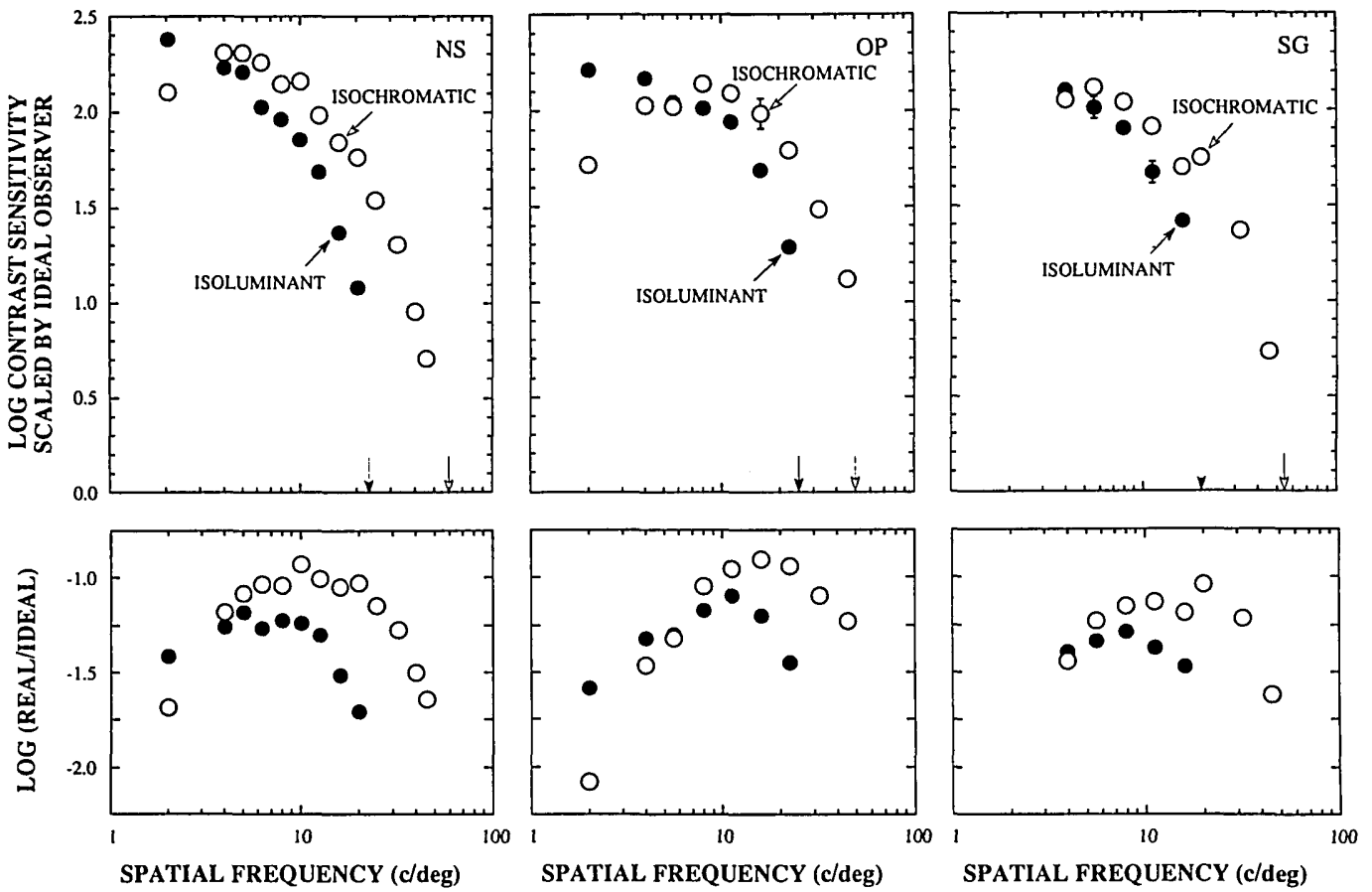

Fig. 9. Top: Foveal contrast-sensitivity functions for isoluminant and isochromatic interference fringes for three observers. Filled and open arrows represent the foveal resolution limit for the isoluminant and the isochromatic stimuli, respectively. Bottom: The ratio of the ideal to the real observer's contrast sensitivity for detecting isoluminant (filled circles) and isochromatic (open circles) stimuli.

The vertical axes in the top panels show log contrast scaled by the results of our ideal-observer analysis, which allows us to plot contrast-sensitivity functions for all our color directions on a single ordinate. In this figure the contrast-sensitivity curves for the red-green isoluminant fringe were shifted $0.28 \log$ unit upward relative to those for the isochromatic fringe. Differences between contrast sensitivities plotted on this ordinate indicate differences in neural efficiency. The red-green isoluminant contrast-sensitivity function declines faster than the isochromatic contrast-sensitivity function.

The bottom panels show the ratio of real- to idealobserver performance explicitly. The results show that the ideal observer is much more sensitive (10-30 times) than the real observer, which is consistent with previous studies for isochromatic stimuli. ${ }^{22}$ If the shape of the contrast-sensitivity function were determined only by optical factors, then the plot of the ratio would not vary with spatial frequency. For both isochromatic and red-green isochromatic stimuli, however, the plot of the ratio has a bell-like shape for all observers.

\section{EXPERIMENT 4: PARAFOVEAL CONTRAST-SENSITIVITY FUNCTIONS}

\section{Methods}

We measured contrast-sensitivity functions in the parafovea for red-green isoluminant and the S-cone-isolated fringes. In a single session all spatial frequencies to be tested were presented in random order. One experimental session lasted approximately $2 \mathrm{~h}$, including two or three intermissions. At least two sessions were run on different days for each observer. The isoluminant and the isochromatic contrast-sensitivity functions were measured with the psychophysical technique. Two color normals (OP and NS) served as observers. We compensated for speckle masking as we had for the foveal measurements.

\section{Results}

The top panels in Fig. 10 show the red-green isoluminant and the S-cone contrast-sensitivity functions for two observers. Error bars represent \pm 1 standard error of mean, although most of them are smaller than the symbols.

As before, the ordinate employs the equivalent contrast metric derived from the ideal observer. The S-coneisolated and the red-green isoluminant contrastsensitivity functions have the same shape. Furthermore, the two functions are superimposed vertically, indicating that these two types of grating are detected by neural mechanisms that have the same efficiency.

The bottom panels of Fig. 10 show the ratio of real- to ideal-observer performance. The ideal observer is much more sensitive (40-100 times) than the real observer. This ratio is higher than that found in the fovea. For both color directions, the plots of the ratio decrease with spatial frequency, suggesting that neural efficiency declines with increasing spatial frequency.

\section{DISCUSSION}

Comparison with Previous Measurements of Isoluminant Contrast Sensitivity

Previous studies of red-green isoluminant contrast sensitivity have attempted to avoid luminance artifacts that are due to chromatic aberrations. Some investigators have used achromatizing lenses to correct the axial chromatic aberration. . $^{15,29,30}$ However, achromatizing lenses do not correct for transverse chromatic aberration and 
chromatic parallax..$^{25,56}$ Noorlander et al..$^{31}$ precisely positioned a 2.5-mm artificial pupil to reduce the effects of chromatic parallax but did not pay special attention to axial chromatic aberration. With a $2.5-\mathrm{mm}$ pupil, the depth of focus is $\sim 0.3 \mathrm{D},{ }^{57}$ which is smaller than the focusing error between red and green lights (e.g., $0.52 \mathrm{D}$ between $520 \mathrm{~nm}$ and $620 \mathrm{~nm}$ ), so this does not completely eliminate the effects of axial chromatic aberration. Granger and Heurtley, ${ }^{16}$ without correcting chromatic aberrations, measured the contrast sensitivity based on color appearance. Their data do not permit an estimate of the neural factors affecting isoluminant contrast sensitivity, because the retinal contrast of their test stimuli is not known.

Mullen ${ }^{17,32}$ and Anderson et $a l^{33}$ had independent control of the parameters defining their red and green gratings. They placed the two gratings at different distances from the observer's eye to compensate for the axial chromatic aberration. Moreover, they allowed the observer to control the two grating spatial frequencies to compensate for the magnification error caused by transverse chromatic aberration. Their results are the most directly comparable with the data obtained in this study.

In Fig. 11 our foveal red-green isoluminant contrastsensitivity functions (filled symbols) are plotted, along with those obtained by Mullen ${ }^{17}$ and Anderson et al..$^{33}$ Different wavelengths were used in the different studies, but we have compensated for this by applying the equivalent contrast metric. Moreover, to compensate for the difference in the retinal illuminance level of the grating stimuli, we shifted the data from Mullen ${ }^{17}$ upward in accordance with the square-root (de Vries-Rose) law. Although the data from previous studies were obtained with larger test stimuli, this is not a problem here because these stimuli are much larger than the grating summation area
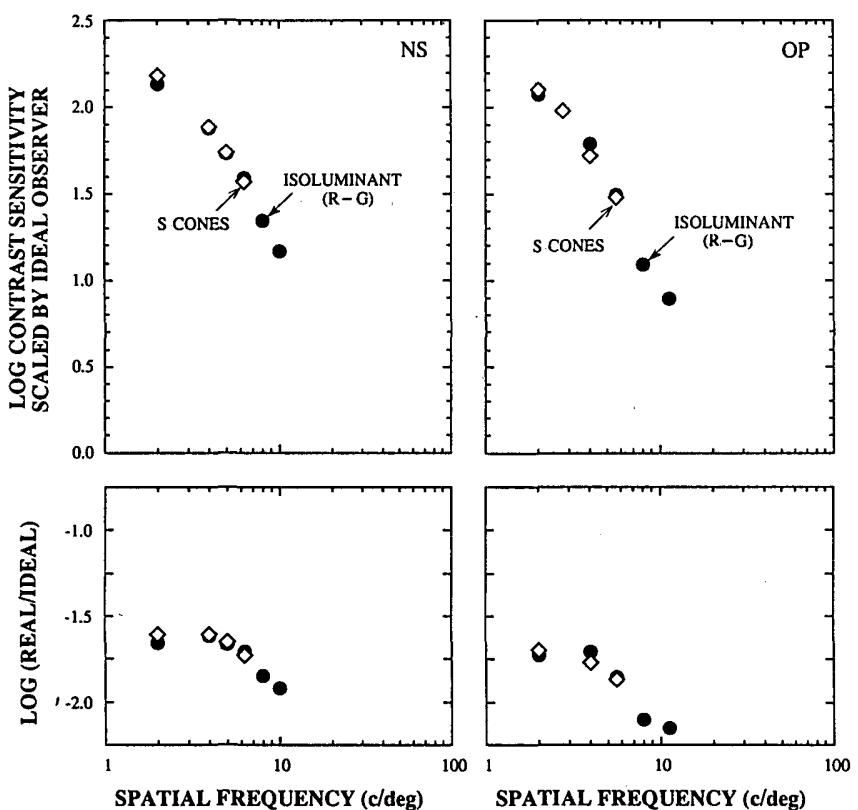

Fig. 10. Top: Parafoveal contrast-sensitivity functions for isoluminant and isochromatic interference fringes for two observers. Bottom: The ratio of the ideal to the real observer's contrast sensitivity for detecting isoluminant (filled circles) and isochromatic (open diamonds) stimuli.

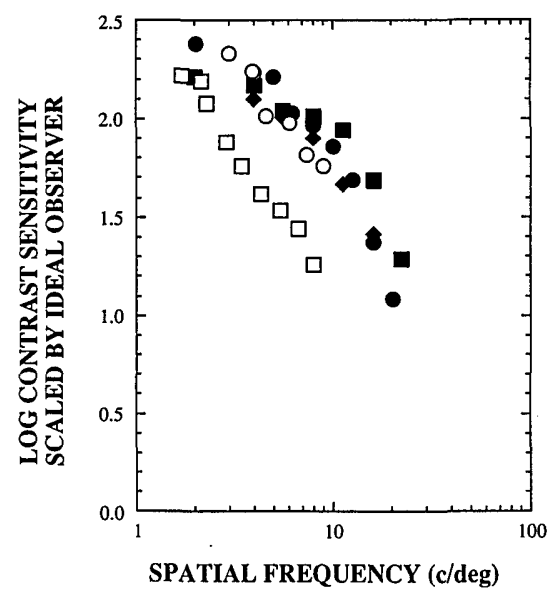

Fig. 11. Comparison of isoluminant red-green contrastsensitivity functions with those obtained previously. Filled symbols represent the foveal data from the three observers measured in this study. Open squares and open circles are the data from Mullen ${ }^{17}$ and Anderson et al., ${ }^{33}$ respectively. The data are plotted with the equivalent contrast metric to compensate for the difference in the stimulus conditions.

for the isoluminant stimuli. Despite similar experimental conditions and techniques, the data from Mullen $^{17}$ and Anderson et $a l^{33}$ differ from each other by $\sim 0.5 \log$ unit at high spatial frequencies. The reasons for this difference are unknown. The data from Anderson et $a l^{33}$ seem to agree with our data, although contrast sensitivity is slightly lower in those of Anderson et al. at the highest spatial frequencies. Presumably this difference is due to optical blurring, which was a factor in their study but not in ours.

\section{Grating Summation Area}

The mechanism underlying the grating summation area is unclear, but it may be explained by the number of contrast detectors across which probability summation provides an improvement in sensitivity ${ }^{58}$ or by the number of cortical neurons from which a central integrator receives converging input. ${ }^{59}$ Whatever the mechanism, the use of a constant number of cycles at all spatial frequencies in our psychophysical measurements prevents the grating summation area from limiting human performance. Since there is no such limitation from the grating summation area in ideal-observer performance, the effect of the grating summation area would have dominated our estimates of neural efficiency if we had used a grating patch of fixed size. Thus the estimates of neural efficiency intentionally exclude the large effects of the grating summation area.

Several previous reports have shown that the detection of isochromatic gratings is improved by an increase in the number of cycles presented up to a critical number of approximately 8-20 cycles, beyond which the contrast sensitivity reaches an asymptotic level..$^{31,58-66}$ Our estimates of the isochromatic summation (5 cycles) area are lower than those of previous studies.

Our results, at the relatively high spatial frequencies that we used, show that the visual system possesses similar grating summation properties for isochromatic, redgreen isoluminant, and S-cone-isolated gratings. This is 

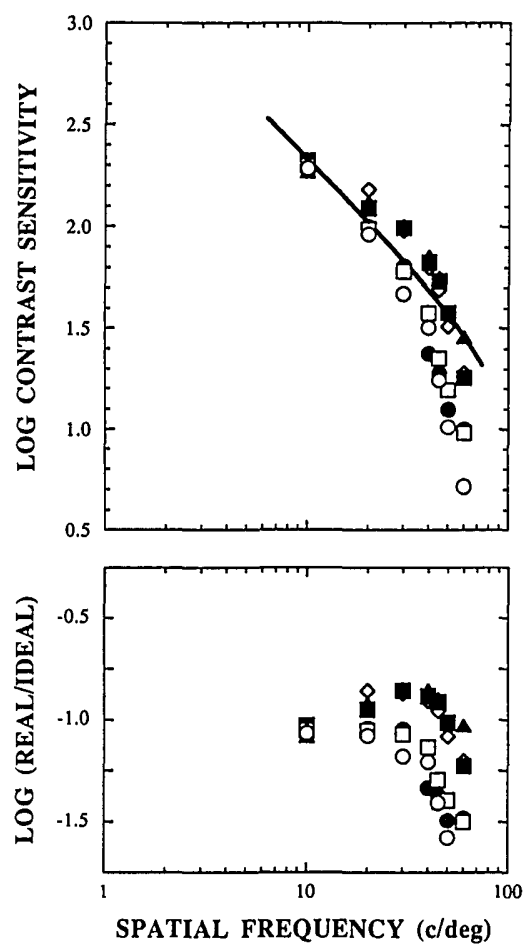

Fig. 12. Top: Comparison of the ideal observer's contrast sensitivity (solid curve) with contrast sensitivities obtained with isochromatic interference fringes. ${ }^{45}$ Bottom: The ratio of the ideal to the real observer's contrast sensitivity.

true at both retinal locations that we tested. Mullen ${ }^{32}$ provides psychophysical estimates of the summation properties for red-green isoluminant modulations at lower spatial frequencies $(0.24-3.2 \mathrm{c} / \mathrm{deg})$. Her estimates of the critical number of cycles are lower than ours (1-3.5 cycles) and may indicate a spatial-frequency dependence of the summation area for red-green isoluminant gratings similar to the dependence that exists for isochromatic gratings.

In our data the grating summation area tends to become less clear in the parafovea, in particular for observer OP. The peripheral data from Robson and Graham's study ${ }^{58}$ also show this tendency. The grating summation area is presumably affected by retinal inhomogeneity. The fovea generally has higher contrast sensitivity at all spatial frequencies, so that increasing field size there eventually encroaches on less-sensitive surrounding retinal regions. Just the opposite is true for fields centered in the parafovea. As their size is increased, they eventually encroach on the more-sensitive fovea. Thus retinal inhomogeneity could reduce the size of the grating summation area in the fovea and enlarge it in the parafovea.

\section{Neural Efficiency for Detecting Isochromatic Gratings}

A major result of this study is that the ideal observer fails to predict the shape of the real observer's isochromatic contrast-sensitivity function at high spatial frequencies. As shown in Fig. 12, previous interferometric data are consistent with this conclusion. In the top panel of Fig. 12 we compare the interferometric contrast-sensitivity functions measured by Williams ${ }^{50}$ with those predicted by our ideal observer. These data were obtained with a fixed field size. To take this into account in generating the ideal-observer prediction, we assumed that the ideal observer had a grating summation area with a diameter inversely proportional to spatial frequency. The different symbols represent the data from different observers in Ref. 50, and the solid curve represents the corresponding ideal observer's contrast-sensitivity function. The bottom panel shows the ratio of the real to the ideal observer's contrast sensitivity. The resulting functions are not flat and resemble the results shown in Figs. 9 and 10. The discrepancy systematically increases with spatial frequency higher than $30 \mathrm{c} / \mathrm{deg}$ and reaches $0.2-0.55$-log-unit difference at $60 \mathrm{c} / \mathrm{deg}$. We considered the possibility that masking by laser speckle might have altered the shape of the real observer's contrast-sensitivity function. Our use of an incoherent background, which is speckle free, was designed to minimize these masking effects. In any case, the effects of speckle masking tend to decline with increasing spatial frequency. ${ }^{50}$ Thus any residual speckle masking would make the measured falloff in contrast sensitivity shallower than it would be in the absence of speckle. This would reduce, not increase, the apparent size of the neural losses that we have identified.

The result that neural efficiency declines somewhat with increasing spatial frequency is inconsistent with the conclusions of Banks et al. ${ }^{22}$ who argued that all the highspatial-frequency falloff in isochromatic contrast sensitivity could be attributed to optical factors. We consider two possible reasons for the difference. One is that our estimate of cone aperture is too small. However, we determined the size of the cone aperture that was necessary in the ideal observer to make neural efficiency independent of spatial frequency. We found that the cone aperture would have to be $\sim 1.5$ times larger than the cone spacing, which is unlikely to be the case. Banks et $a l .{ }^{22}$ assumed that the diameter of the cone aperture is equal to the cone spacing, which is probably too large. ${ }^{36,67}$ This would steepen the ideal observer's falloff of contrast sensitivity at high spatial frequencies and tend to hide a loss in neural efficiency. A second possible reason is that Banks et al. may have used estimates of the optical quality of the human eye that were too low. This would also steepen the falloff of their ideal observer's contrast sensitivity. An advantage of our study is that we do not require any assumptions about the optical quality of the eye's dioptrics.

Chen $e t a l^{68}$ support our view with an entirely different experimental method. They measured contrastsensitivity functions for distortion products obtained by the addition of two interference fringes of different spatial frequency or orientation. Observers perceive the distortion product as much coarser stripes than the original fringes because of a nonlinearity of the visual system that presumably exists in the early retina. Their contrastsensitivity data also show falloffs at high spatial frequencies, suggesting the presence of neural blurring.

\section{Relative Neural Efficiency for Detecting Red-Green Isoluminant and S-Cone Isolated Gratings}

A major result of this study is that when we plot contrast sensitivity using an ordinate that takes optical factors into account, the contrast-sensitivity functions for red-greenisoluminant and S-cone-isolated fringes have the same shape and vertical position (see Fig. 10). The agreement is surprisingly good, especially given that the ideal- 
observer parameters were taken from the literature and were not adjusted to bring the two curves into alignment. The agreement implies that the neural mechanisms responsible for detecting stimuli in these two color directions have similar sensitivity and spatial properties. If performance is limited by the structure of the receptive fields, then our result predicts that the receptive fields of units tuned for red-green isoluminant and S-coneisolated stimuli will have similar spatial profiles. The difference seen between red-green isoluminant and Scone-isolated gratings when contrast sensitivity is plotted in a conventional manner can be explained primarily by the low photon catch of the sparse S-cone submosaic. This effect is tempered by the spectral overlap of the Land the $\mathrm{M}$-cone responsivities that affect red-green isoluminant contrast sensitivity.

Despite this result, we are not arguing that the resolution limits for red-green isoluminant and S-cone-isolated gratings are the same. Our results from this study and from the companion paper suggest that the resolution limit for red-green isoluminant stimuli is $20-27 \mathrm{c} / \mathrm{deg}{ }^{26}$ Measurements of the resolution limit for S-cone-isolated stimuli are typically $\sim 10 \mathrm{c} / \mathrm{deg}$ and never exceed $14 \mathrm{c} / \mathrm{deg}{ }^{46,47}$ Resolution for S-cone stimuli is limited by ambiguity that arises because of the low sampling rate of the S-cone submosaic. ${ }^{46,47}$ The corresponding sampling limits for red-green isoluminant stimuli are higher. The ideal-observer analysis shows that spatial sampling by the cone mosaic does not influence the shape of the contrastsensitivity function. Spatial sampling does affect the vertical position of the contrast-sensitivity function through its influence on photon catch. These results are consistent with theoretical arguments ${ }^{47,69}$ that spatial sampling does affect the contrast-sensitivity functions of real observers in the spatial-frequency range where gratings can be resolved. Above this range, aliasing by the cone mosaic can influence the shape of real observers' contrast-sensitivity functions, ${ }^{70}$ because aliasing changes the spatial-frequency content of the stimulus and because neural efficiency for real observers depends on spatial frequency.

\section{Relative Neural Efficiency for Detecting Isochromatic and Isoluminant Gratings}

Our results show that the neural efficiency for detecting isoluminant gratings falls more steeply with increasing spatial frequency than that for isochromatic gratings. Because we have taken care to remove optical factors, such as chromatic aberration, from this comparison, the overlap in the L- and the M-cone spectral responsivities and the scarcity of $\mathrm{S}$ cones cannot account for this difference. The difference must be due to neural factors. There are two qualitatively different ways to explain the difference in neural efficiency for detecting isochromatic and isoluminant gratings. One possibility is that there is an additional sensitivity loss for isoluminant stimuli in the visual system that does not depend on spatial frequency. In this case an additional vertical shift of the isoluminant contrast-sensitivity functions would be expected to provide a good fit to the isochromatic contrast-sensitivity function. However, more than 1 log unit of vertical shift is required for bringing the isoluminant contrast-sensitivity functions into alignment at high spatial frequencies, and then the functions do not overlap at moderate spatial frequencies. Furthermore, this magnitude of vertical shift pushes the red-green-isoluminant contrast-sensitivity function above the theoretical limit imposed by the idealobserver contrast-sensitivity function. Although we cannot rule out some spatial-frequency-independent sensitivity loss, such a loss alone cannot explain the difference in neural efficiency for isochromatic and isoluminant stimuli.

A second possibility is that there is a difference in spatial bandwidth of the neural visual system for isoluminant and for isochromatic stimuli. In this case, a horizontal shift of the isoluminant contrast-sensitivity functions should permit a good fit with the isochromatic contrastsensitivity function at high spatial frequencies. Figure 13 shows the fit between the foveal isochromatic and the redgreen isoluminant contrast-sensitivity functions after the isoluminant function has been shifted horizontally $0.25 \mathrm{log}$ unit (factor of 1.8). The fit is good at moderate and high spatial frequencies. At low frequencies the fit is not good because of the well-known bandpass character of isochromatic contrast sensitivity. The same shift was required for all three observers. The good agreement at moderate and high spatial frequencies suggests that the difference between isochromatic and isoluminant contrast sensitivity is due to a difference in spatial bandwidth rather than to a loss of sensitivity that is independent of spatial frequency. At low temporal frequencies where our measurements were made, the spatial bandwidth of the neural visual system for isochromatic stimuli is roughly twice that for isoluminant stimuli.

\section{Neural Efficiency for Isoluminant Stimuli at Low Spatial Frequencies}

Despite the fact that the isoluminant contrast-sensitivity functions decline monotonically with increasing spatial frequency, their corresponding neural-efficiency functions initially rise and then decline. This low-frequency cut in neural efficiency is consistent with other psychophysical results. $^{71}$ It may be due to a decline in the number of cycles in the summation area at low spatial frequencies. Alternatively, it may reveal the action of multiplicative or subtractive mechanisms of chromatic adaptation. The magnitude of the low-frequency cut is smaller for isoluminant stimuli than for isochromatic stimuli, and this may reflect different demands on the adaptive mechanisms that protect the visual system from saturation. Mechanisms that are sensitive to isochromatic stimuli must cope with a huge dynamic range of overall light intensities in the visual environment. These changes in light intensity generate small changes in the differences among the outputs of cones (i.e., chromatic signals). Consequently, the amount of chromatic adaptation required for avoiding saturation should be less for isoluminant than for isochromatic stimuli. ${ }^{72}$

Chaparro et $a l .^{73}$ have recently reported that color is what the eye sees best. This conclusion was based on asking what threshold stimulus has the smallest contrast energy. Our neural-efficiency measure captures a similar notion of how well the eye sees. Our efficiency measurements do show a superiority for isoluminant stimuli at low spatial frequencies. Maximum neural efficiencies in our experiment, however, occur for isochromatic stimuli 

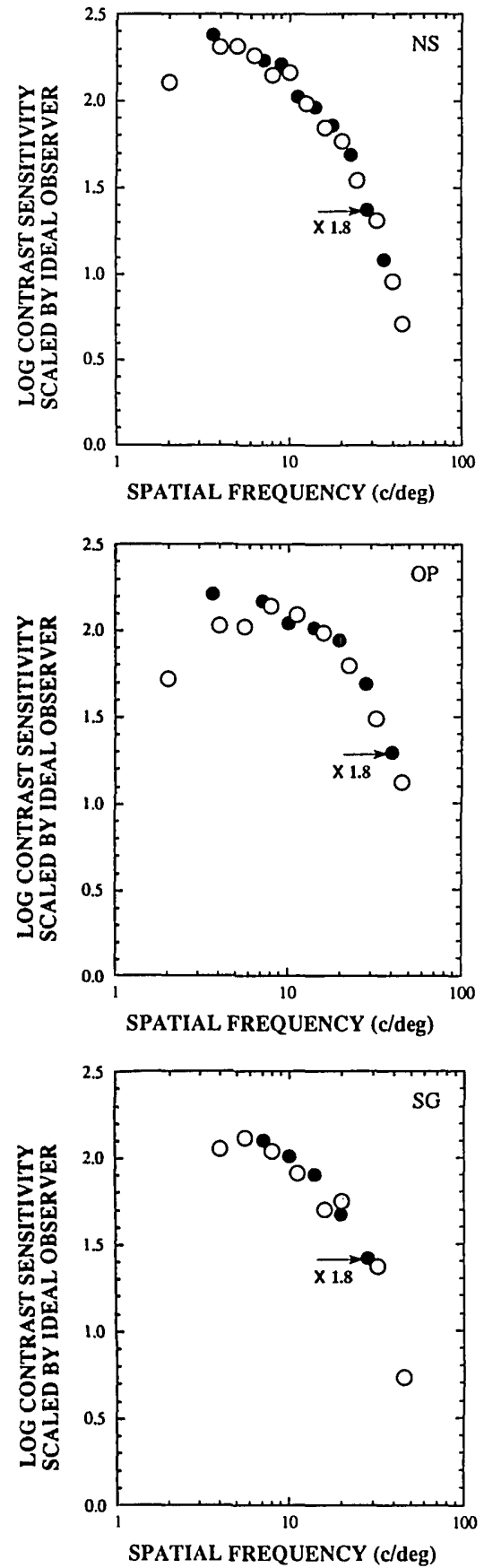

Fig. 13. Isoluminant contrast sensitivities are shifted laterally by $0.25 \log$ unit (a factor of 1.8) for the best fit of isochromatic contrast sensitivity.

at moderate spatial frequencies in all three observers. Direct comparison of the two sets of results is hampered by the fact Chaparro et al. made their measurements with small circular test flashes, while we used extended grating stimuli.

\section{Spatial-Filtering Model of Neural Efficiency for Different} Color Directions

In this subsection we assume that the changes in neural efficiency that we have observed are the result of a spatial filter (the bandwidth of which depends on color direction) followed by additive spatial-frequency-independent noise. We then ask what properties this spatial filter would have to have in order to account for our neural efficiencies.
We restrict our analysis to the neural-efficiency estimates at high spatial frequencies, avoiding complications introduced by the bandpass nature of the isochromatic contrastsensitivity function and by uncertainty about the grating summation area at low spatial frequencies. If this hypothetical spatial filter were implemented with a neural point spread, then our analysis could be interpreted as examining the spatial profile of the neural receptive field center, which dominates performance at high spatial frequencies.

To calculate the spatial-filter properties, we fitted a Gaussian function to the high-frequency portion of the ratio between real and ideal contrast sensitivity in the fovea. We then computed the Fourier transform of this Gaussian to obtain the profile of a spatial filter that could account for our results. The results of this calculation are shown in Fig. 14. The bottom row of the figure shows the point-spread function of the dioptrics of the eye. ${ }^{57}$ The next row up shows the row spacing of the cones, $0.54 \mathrm{~min}$ in the fovea and $0.95 \mathrm{~min}$ in the parafovea. ${ }^{70,74,75}$ The Gaussian functions that appear immediately above the cones represent the profiles of foveal cone apertures, the full widths at half-height of which are $0.23 \mathrm{~min}$ in the fovea and $0.40 \mathrm{~min}$ in the parafovea. ${ }^{37}$ The Gaussian functions in the top three rows represent the profiles of the estimated neural point spread for the isochromatic, the red-green isoluminant, and the S-cone-isolated gratings. In the fovea the size of the neural point spread, in terms of full width at half-height, is $0.71 \mathrm{~min}$ for isochromatic gratings and $1.53 \mathrm{~min}$ for red-green-isoluminant gratings. These are 3.1 and 6.8 times larger, respectively, than cone-aperture size at the foveal center. Note that the ratio of the computed neural point-spread sizes (2.15) is slightly greater than the measured bandwidth difference (factor of 1.8) between isochromatic and isoluminant stimuli. This is because the bandwidth difference includes the effects of blurring by the cone aperture as well as the subsequent neural blurring. At an eccentricity of $1 \mathrm{deg}$, the size of the neural point spread is $2.89 \mathrm{~min}$ for both red-green isoluminant stimuli and S-cone-isolated
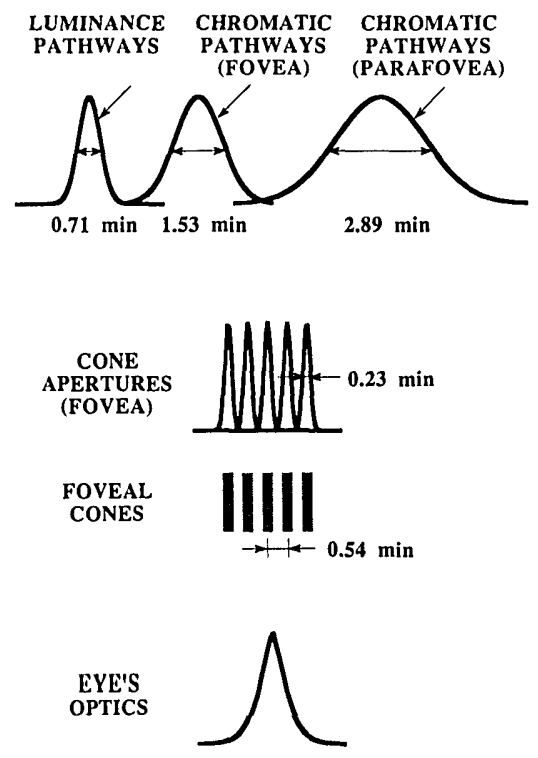

Fig. 14. Comparison of the size of the neural point spread for chromatic and luminance pathways with those of the pointspread function of the eye's optics, cone aperture, and cone spacing at the fovea. 
stimuli. This is 7.2 times larger than the parafoveal cone-aperture size.

The neural point spread for the isochromatic gratings is somewhat larger than the area of a single cone. Although this point-spread function must have a neural origin, it is comparable in size with the point-spread function of the eye's dioptrics. ${ }^{57}$ It makes sense that the optical and the neural blurring are similar, since there would be little evolutionary pressure for one to be considerably different from the other. If there were a difference, it would not result in much improvement in performance. The physiological mechanism that produces this neural blurring is not known. If ganglion-cell receptive-field centers receive input only from single cones, ${ }^{76-80}$ then the fact that the neural point-spread function is larger than the area of a single cone means that the neural blur must occur at a central site. However, the functional connectivity of cones to retinal ganglion cells is not well enough known to permit us to exclude the possibility that neural blurring occurs as a result of lateral connections in the retina.

Recently Chen et $a l .{ }^{68}$ reported slightly larger estimates for the neural point spread $(0.9 \mathrm{~min}$ in terms of full width at half-height) than ours $(0.7 \mathrm{~min})$. They obtained estimates with the contrast sensitivity for the nonlinear distortion product without removing the effect of grating summation area. Since their point spread was calculated based on a deviation between the contrast sensitivity and a constant value (i.e., slope of 0 ), their estimates would be larger than those calculated with a slope of -1 , as in the case of fixed number of grating cycles. (From our calculation, the point-spread function obtained based on a slope of 0 provides roughly 1.4 times larger estimates than that based on a slope of -1 .) Other factors to be considered are relatively large individual differences in contrast sensitivity, as reported by Williams. ${ }^{50}$ Moreover, in the study of Chen et al. ${ }^{68}$ perhaps the size of the distortion product was not fixed, because the visible area of the distortion product usually decreases with increasing spatial frequency. Considering these factors, we can say that there is reasonable agreement between the two studies on the estimates of neural point-spread function.

The neural point-spread function for the red-greenisoluminant gratings covers an area that contains an even larger number of cones. The S-cone signals are also processed with a more-or-less similar neural point-spread function. For S-cone-isolated stimuli, however, the sparse number of $S$ cones (5-10\% of total cone population ${ }^{37,51-53}$ ) means that no more than two cones could feed the center to any substantial extent.

Models have been proposed by Ingling and Martinez, ${ }^{81}$ Rohaly and Buchsbaum, ${ }^{82,83} \mathrm{Kelly}^{84}$ and Ingling ${ }^{85}$ to provide accounts of the difference between isochromatic (bandpass) and isoluminant (low-pass) contrast sensitivity at low spatial frequencies in terms of the properties of center-surround units. However, these models in their current forms do not predict the difference in neural bandwidth that we have observed at high spatial frequencies.

\section{ACKNOWLEDGMENTS}

We are grateful to Wilson Geisler and Martin Banks, who provided source programs of their ideal-observer model and valuable suggestions on this study; to Christine Curcio, who provided the cone mosaic data and useful comments; to Ken Sloan, who provided source programs for triangulation analysis; to Hugh Perry, who provided the foveal cone mosaic data; and to Orin Packer and Susan Galvin, who served as observers. We also thank Bill Haake and Alan Russell for their technical assistance. This research was supported by grants EY04367, EY01319, EY06278, and EY09625 from the National Institutes of Health.

*Present address, Basic Research Department, Olympus Optical Co., Ltd., 2-3 Kuboyama, Hachioji, Tokyo 192 Japan.

${ }^{\dagger}$ Present address, Department of Psychology, University of California, Santa Barbara, Santa Barbara, California 93106.

\section{REFERENCES}

1. C. Lu and D. H. Fender, "The interaction of color and luminance in stereoscopic vision," Invest. Ophthalmol. Vis. Sci. 11, 482-489 (1972).

2. C. M. M. De Weert and K. J. Sadza, "New data concerning the contribution of colour differences to stereopsis," in Colour Vision, J. D. Mollon and L. T. Sharpe, eds. (Academic, London, 1983), pp. 553-562.

3. D. L. Grinberg and D. R. Williams, "Stereopsis with chromatic signals from the blue-sensitive mechanism," Vision Res. 25, 531-537 (1985).

4. $\mathrm{H}$. De Lange, "Research into the dynamic nature of the human fovea-cortex systems with intermittent and modulated light. II. Phase shift in brightness and delay in color perception," J. Opt. Soc. Am. 48, 784-789 (1958).

5. J. Krauskopf and D. R. Williams, "Temporal frequency response of chromatic and luminance mechanisms," Invest. Ophthalmol. Vis. Sci. Suppl. 20, 61 (1981).

6. V. S. Ramachandran and R. L. Gregory, "Does colour provide an input to human motion perception?" Nature (London) 275, 55-56 (1978).

7. P. Cavanagh, C. W. Tyler, and O. E. Favreau, "Perceived velocity of moving chromatic gratings," J. Opt. Soc. Am. A 1, 893-899 (1984).

8. D. T. Lindsey and D. Y. Teller, "Motion at isoluminance: discrimination/detection ratios for moving isoluminant gratings," Vision Res. 30, 1751-1761 (1990).

9. J. M. Wolfe and D. A. Owens, "Is accommodation colorblind? Focusing chromatic contours," Perception 10, 53-62 (1981).

10. E. Switkes, A. Bradley, and C. Schor, "Readily visible changes in color contrast are insufficient to stimulate accommodation," Vision Res. 30, 1367-1376 (1990).

11. R. M. Boynton, "Ten years of research with the minimally distinct border," in Visual Psychophysics and Physiology, J. C. Armington, J. Krauskopf, and B. R. Wooten, eds. (Academic, New York, 1978), pp. 193-207.

12. B. W. Tansley and R. M. Boynton, "Chromatic border perception: the role of red- and green-sensitive cones," Vision Res. 18, 683-697 (1978).

13. M. J. Morgan and T. S. Aiba, "Positional acuity with chromatic stimuli," Vision Res. 25, 689-695 (1985).

14. J. Krauskopf and B. Farell, "Vernier acuity: effects of chromatic content, blur and contrast," Vision Res. 31, 735-749 (1991).

15. R. Hilz and C. R. Cavonius, "Wavelength discrimination measured with square-wave gratings," J. Opt. Soc. Am. 60, 273-277 (1970).

16. E. M. Granger and J. C. Heurtley, "Visual chromaticitymodulation transfer function," J. Opt. Soc. Am. 63, 11731174 (1973).

17. K. T. Mullen, "The contrast sensitivity of human color vision to red-green and blue-yellow chromatic gratings," J. Physiol. 359, 381-400 (1985).

18. W. S. Geisler, "Sequential ideal-observer analysis of visual discriminations," Psychol. Rev. 96, 267-314 (1989).

19. J. R. Jordan III, W. S. Geisler, and A. C. Bovik, "Color as a source of information in the stereo correspondence process," Vision Res. 30, 1955-1970 (1990). 
20. W. S. Geisler, "Physical limits of acuity and hyperacuity," J. Opt. Soc. Am. A 1, 775-782 (1984).

21. W. S. Geisler and K. D. Davila, "Ideal discriminators in spatial vision: two-point stimuli," J. Opt. Soc. Am. A 2, 14831497 (1985).

22. M. S. Banks, W. S. Geisler, and P. J. Bennett, "The physical limits of grating visibility," Vision Res. 27, 1915-1924 (1987)

23. M. S. Banks and P. J. Bennett, "Optical and photoreceptor immaturities limit the spatial and chromatic vision of human neonates," J. Opt. Soc. Am. 5, 2059-2079 (1988).

24. L. N. Thibos, A. Bradley, and D. L. Still, "Interferometric measurement of visual acuity and the effect of ocular chromatic aberration," Appl. Opt. 30, 2079-2087 (1991).

25. F. W. Campbell and R. W. Gubisch, "The effect of chromatic aberration on visual acuity," J. Physiol. 192, 345-358 (1967)

26. N. Sekiguchi, D. R. Williams, and D. H. Brainard, "Aberration-free measurements of the visibility of isoluminant gratings," J. Opt. Soc. Am. A 10, 2105-2117 (1993).

27. H. C. Howland and B. Howland, "A subjective method for the measurement of monochromatic aberrations of the eye," J. Opt. Soc. Am. 67, 1508-1518 (1977).

28. O. H. Schade, "On the quality of color-television images and the perception of color detail," J. Soc. Motion Pict. Telev. Eng. 67, 801-819 (1958).

29. G. J. C. Van der Horst and M. A. Boumann, "Spatiotemporal chromaticity discrimination," J. Opt. Soc. Am. 59, 14821488 (1969).

30. G. J. C. Van der Horst, C. M. M. De Weert, and M. A. Boumann, "Transfer of spatial chromaticity-contrast at threshold in the human eye," J. Opt. Soc. Am. 57, 1260-1266 (1967).

31. C. Noorlander, M. J. G. Heuts, and J. J. Koenderink, "Influence of the target size on the detection threshold for luminance and chromaticity contrast," J. Opt. Soc. Am. 70, 1116-1121 (1980).

32. K. T. Mullen, "Colour vision as a post-receptoral specialization of the central visual field," Vision Res. 31, 119-130 (1991).

33. S. J. Anderson, K. T. Mullen, and R. F. Hess, "Human peripheral spatial resolution for achromatic and chromatic stimuli: limits imposed by optical and retinal factors," J. Physiol. 442, 47-64 (1991).

34. G. Wyszecki and W. S. Stiles, Color Science (Wiley, New York, 1982).

35. J. M. Enoch and F. L. Tobey, Jr., "Waveguide properties of retinal receptors: techniques and observations," in Vertebrate Photoreceptor Optics, J. M. Enoch and F. L. Tobey, Jr., eds. (Springer-Verlag, New York, 1981), pp. 169-218.

36. D. I. A. MacLeod, D. R. Williams, and W. Makous, "A visual nonlinearity fed by single cones," Vision Res. 32, 347-363 (1992).

37. C. A. Curcio, K. A. Allen, K. R. Sloan, C. L. Lerea, J. B. Hurley, I. B. Klock, and A. H. Milam, "Distribution and morphology of human cone photoreceptors stained with anti-blue opsin," J. Comp. Neurol. 312, 610-624 (1991).

38. D. R. Williams, D. I. A. MacLeod, and M. M. Hayhoe, "Foveal tritanopia," Vision Res. 21, 1341-1356 (1981).

39. J. J. Vos and P. L. Walraven, "On the deviation of the foveal receptor primaries," Vision Res. 11, 799-818 (1971).

40. P. L. Walraven, "A closer look at the tritanopic convergence point," Vision Res. 14, 1339-1343 (1974).

41. V. C. Smith and J. Pokorny, "Spectral sensitivity of the foveal cone pigments between 400 and $500 \mathrm{~nm}$," Vision Res. 15, 161-171 (1975).

42. R. L. P. Vimal, J. Pokorny, V. C. Smith, and S. K. Shevell, "Foveal cone thresholds," Vision Res. 29, 61-78 (1989).

43. C. M. Cicerone and J. L. Nerger, "The relative numbers of long-wavelength-sensitive to middle-wavelength-sensitive cones in the human fovea centralis," Vision Res. 29, 115-128 (1989).

44. D. A. Baylor, B. J. Nunn, and J. L. Schnapf, "Spectral sensitivity of cones of the monkey Macaca fascicularis," J. Physiol. 390, 145-160 (1987).

45. N. Sekiguchi, "Contrast sensitivity for isoluminant interference fringes in human foveal vision," Ph.D. dissertation (University of Rochester, Rochester, N.Y., 1992).

46. D. R. Williams and R. Collier, "Consequences of spatial sampling by a human photoreceptor mosaic," Science 221, 385-387 (1983).
47. D. R. Williams, R. J. Collier, and B. J. Thompson, "Spatial resolution of the short-wavelength mechanism," in Colour Vision, J. D. Mollon and L. T. Sharpe, eds. (Academic, London, 1983), pp. 487-503.

48. F. W. Campbell, R. H. S. Carpenter, and J. Z. Levinson, "Visibility of aperiodic patterns compared with that of sinusoidal gratings," J. Physiol. 204, 283-298 (1969).

49. D. H. Kelly, "Effects of sharp edges on the visibility of sinusoidal gratings," J. Opt. Soc. Am. 60, 98-103 (1970).

50. D. R. Williams, "Visibility of interference fringes near the resolution limit," J. Opt. Soc. Am. A 2, 1087-1093 (1985).

51. R. E. Marc and H. G. Sperling, "Chromatic organization of primate cones," Science 196, 454-456 (1977).

52. F. M. De Monasterio, E. P. McCrane, J. K. Newlander, and S. J. Schein, "Density profile of blue-sensitive cones along the horizontal meridian of macaque retina," Invest. Ophthalmol. Vis. Sci. 26, 289-302 (1985).

53. P. K. Ahnelt, H. Kolb, and R. Pflug, "Identification of a subtype of cone photoreceptor, likely to be blue-sensitive, in the human retina," J. Comp. Neurol. 255, 18-34 (1987).

54. F. L. Van Nes and M. A. Bouman, "Spatial modulation transfer in the human eye," J. Opt. Soc. Am. 57, 401-406 (1967).

55. D. H. Kelly, "Adaptation effects on spatio-temporal sine-wave thresholds," Vision Res. 12, 89-101 (1972).

56. X. Zhang, A. Bradley, and L. N. Thibos, "Achromatizing the human eye: the problem of chromatic parallax," J. Opt. Soc. Am. A 8, 686-691 (1991).

57. G. Westheimer, "The eye as an optical instrument," in Handbook of Perception and Human Performance. Vol. 1. Sensory Processes and Perception. K. R. Boff, L. Kaufman, and J. P. Thomas, eds. (Wiley, New York, 1986), pp. 4.1-4.20.

58. J. G. Robson and N. Graham, "Probability summation and regional variation in contrast sensitivity across the visual field," Vision Res. 21, 409-418 (1981).

59. V. Virsu and J. Rovamo, "Visual resolution, contrast sensitivity, and the cortical magnification factor," Exp. Brain Res. 37, 475-494 (1979).

60. J. M. Findlay, "A spatial integration effect in visual acuity," Vision Res. 9, 157-166 (1969).

61. J. Hoekstra, D. P. J. Van der Goot, G. Van den Brink, and F. A. Bilsen, "The influence of the number of cycles upon the visual contrast threshold for spatial sine wave patterns," Vision Res. 14, 365-368 (1974).

62. O. Estévez and C. R. Cavonius, "Low-frequency attenuation in the detection of gratings: sorting out the artefacts," Vision Res. 16, 497-500 (1976).

63. R. L. Savoy and J. J. McCann, "Visibility of low-spatialfrequency sine-wave targets: dependence on number of cycles," J. Opt. Soc. Am. 65, 343-350 (1975).

64. E. R. Howell and R. F. Hess, "The functional area for summation to threshold for sinusoidal gratings," Vision Res. 18, 369-374 (1978)

65. J. J. Koenderink and A. J. Van Doorn, "Spatial summation for complex bar patterns," Vision Res. 20, 169-176 (1980).

66. J. S. Tootle and M. A. Berkley, "Contrast sensitivity for vertically and obliquely oriented gratings as a function of grating area," Vision Res. 23, 907-910 (1983).

67. W. H. Miller and G. D. Bernard, "Averaging over the foveal receptor aperture curtails aliasing," Vision Res. 23, 13651369 (1983).

68. B. Chen, W. Makous, and D. R. Williams, "Serial spatial filters in vision," Vision Res. 33, 413-427 (1993).

69. J. I. Yellott, Jr., "Spectral consequences of photoreceptor sampling in the rhesus monkey," Science 221, 382-385 (1983).

70. D. R. Williams, "Aliasing in human foveal vision," Vision Res. 25, 195-205 (1985).

71. N. W. Daw, "Visual responses to gradients of varying colour and equal luminance" Nature (London) 203, 215-216 (1964).

72. M. Hayhoe and P. Wenderoth, "Adaptation mechanisms in color and brightness," in From Pigments to Perception, B. B. Lee and A. Valberg, eds. (Plenum, New York, 1991), pp. 353367.

73. A. Chaparro, C. F. Stromeyer III, E. P. Huang, R. E. Kronauer, and R. T. Eskew, Jr., "Colour is what the eye sees best," Nature (London) 361, 348-350 (1993).

74. D. R. Williams, "Topography of the foveal cone mosaic in the living human eye," Vision Res. 28, 433-454 (1988). 
74. D. R. Williams, "Topography of the foveal cone mosaic in the living human eye," Vision Res. 28, 433-454 (1988).

75. N. Sekiguchi, D. R. Williams, and O. Packer, "Nonlinear distortion of gratings at the foveal resolution limit," Vision Res. 31, 815-831 (1991).

76. B. B. Boycott and J. E. Dowling, "Organization of the primate retina: light microscopy," Philos. Trans. R. Soc. London Ser. B 225, 109-184 (1969).

77. H. Kolb, "Organization of the outer plexiform layer of the primate retina: electron microscopy of Golgi-impregnated cells," Philos. Trans. R. Soc. London Ser. B 258, 261-283 (1970).

78. S. J. Schein, "Anatomy of macaque fovea and spatial densities of neurons in foveal representation," J. Comp. Neurol. 269, 479-505 (1988).

79. H. Wässle, U. Grünert, J. Röhrenbeck, and B. B. Boycott, "Cortical magnification factor and the ganglion cell density of the primate retina," Nature (London) 341, 643-646 (1989).
80. H. Wässle, U. Grünert, J. Röhrenbeck, and B. B. Boycott, "Retinal ganglion cell density and cortical magnification factor in the primate," Vision Res. 30, 1897-1911 (1990).

81. C. R. Ingling, Jr., and E. Martinez, "The spatiochromatic signal of the $\mathrm{r}-\mathrm{g}$ channel," in Colour Vision, J. D. Mollon and L. T. Sharpe, eds. (Academic, London, 1983), pp. 433-444.

82. A. M. Rohaly and G. Buchsbaum, "Inference of global spatiochromatic mechanisms from contrast sensitivity functions," J. Opt. Soc. Am. A 5, 572-576 (1988).

83. A. M. Rohaly and G. Buchsbaum, "Global spatiochromatic mechanism accounting for luminance variations in contrast sensitivity functions," J. Opt. Soc. Am. A 6, 312-317 (1989).

84. D. H. Kelly, "Opponent-color receptive field profiles determined from large-area psychophysical measurements," J. Opt. Soc. Am. A 6, 1784-1793 (1989).

85. C. R. Ingling, Jr., "Psychophysical correlates of parvo channel function," in From Pigments to Perception, A. Valberg and B. B. Lee, eds. (Plenum, New York, 1991), pp. 413-424. 